\title{
Reconstruction of the cosmic equation of state for high redshift
}

\author{
A. M. Velasquez-Toribio ${ }^{1, a}$, M. M. Machado ${ }^{1, b}$, Julio C. Fabris ${ }^{1,2, c}$ \\ ${ }^{1}$ Departamento de Física, Nucleo Cosmo-ufes, Universidade Federal do Espirito Santo, Vitǿria, ES 29075-910, Brasil \\ ${ }^{2}$ National Research Nuclear University MEPhI, Kashirskoe sh. 31, Moscow 115409, Russia
}

Received: 14 May 2019 / Accepted: 23 November 2019 / Published online: 13 December 2019

(C) The Author(s) 2019

\begin{abstract}
We investigate the possibilities of reconstructing the cosmic equation of state (EoS) for high redshift. In order to obtain general results, we use two model-independent approaches. The first reconstructs the EoS using comoving distance and the second makes use of the Hubble parameter data. To implement the first method, we use a recent set of Gamma-Ray Bursts (GRBs) measures. To implement the second method, we generate simulated data using the SandageLoeb ( $S L$ ) effect; for the fiducial model, we use the $\Lambda C D M$ model. In both cases, the statistical analysis is conducted through the Gaussian processes (non-parametric). In general, we demonstrate that this methodology for reconstructing the EoS using a non-parametric method plus a modelindependent approach works appropriately due to the feasibility of calculation and the ease of introducing a priori information $\left(H_{0}\right.$ and $\left.\Omega_{m 0}\right)$. In the near future, following this methodology with a higher number of high quality data will help obtain strong restrictions for the EoS.
\end{abstract}

\section{Introduction}

The accelerated expansion of the universe is one of the biggest problems of cosmology today. Initially, it was associated with a cosmological constant or vacuum energy and, subsequently, models with scalar fields (also known as quintessence models) were evoked. Other possibilities include modified gravitation, extra dimensions, and so on. For a recent review, see references [1-3].

On the other hand, among the different cosmological observables, the cosmic equation of state $(\mathrm{EoS})$ is of fundamental importance, as it carries the kinematic and dynamic information of a given cosmological model. The reconstruction of these observables has been widely considered in the

\footnotetext{
a e-mail: alan.toribio@ufes.br

b e-mail: marlonmoro92@googlemail.com

ce-mail: julio.fabris@cosmo-ufes.org
}

literature using different types of cosmological data, such as the following: Supernovae Ia, cosmic background radiation, clusters of galaxies, baryon acoustic oscillations $(B A O)$, Hubble parameter data, $f \sigma_{8}$, and so on. Additionally, different statistical reconstruction techniques have been used [4-10].

Nevertheless, the reconstruction of this observable has not been considered for high redshift, in principle, due to the lack of data for any redshift greater than 2.0. However, this question is currently changing and we can consider the reconstruction of the $\operatorname{EoS}(w(z))$ for high redshifts. Understanding in detail how $w(z)$ evolves as a function of time is fundamental to know the nature of dark energy. For instance, to answer the question if the accelerated expansion is consistent with a local inhomogeneous effect or if the accelerated expansion is a phase of the evolution of the whole universe, we must to know in detail the form of the EoS.

In this paper we reconstruct $w(z)$ using two modelindependent approaches proposed in the literature [11-14]. Specifically, these approximations are given by equations (6) and (17). The reconstruction of the EoS is not completely independent of the model because we need to assume explicit values of $\Omega_{m 0}$ and $H_{0}$. In general, there is no way, in cosmology, to determine the EoS in a completely model-independent way. However, the choice of an adequate statistical method can help to enhance the model-independent approach. In that regard, we use the method of the Gaussian process as a statistical method, which is a non-parametric method and does not require knowing a specific parametric form of the EoS. This statistical approach allows us to get closer to the ideal of a reconstruction that is completely independent from the model.

If the EoS can be determined with high precision, then we can discriminate between different types of cosmological models. However, in the present paper, due to the quality and quantity of the current data, we do not get strong constraints to discriminate between models. Nevertheless, we prove that it is possible to use measured and simulated data to reconstruct 
$w(z)$ for high redshift. The main message of the article is that, even with the inconclusive estimates with the current data, our proposed methodology may be very useful for future data provided by some ongoing projects, such as the Euclid or the LSST among others. ${ }^{1}$

Our paper is organized as follows. In Sect. 2, we present the two reconstruction methods of the EoS and the Gaussian processes. Section 3 is devoted to our results and in the Sect. 4 , we present the conclusions of our study.

\section{Reconstruction of equation of state}

We use two methods to reconstruct the EoS: the first method makes use of distance measurements from Gamma-Ray Bursts (GRBs) and the second uses simulated data of the Hubble parameter generated by the Sandage-Loeb (SL) effect. In this manner, we can form a reconstruction based on observed data and another on simulated data. Both methods allow to reconstruct the EoS using the model-independent approach. Below, we explain in detail each of the methods.

\subsection{The distance modulus of GRBs}

In this case, we are going to reconstruct the EoS using the distance module measures derived from the GRBs. Much research has been conducted to establish GRBs as standard candles, since there is not only one method to standardize GRBs, for example, the distance calibrations GRBs usually make use of some of the empirical luminosity correlations, such as, $\tau_{\text {lag }}-L, V-L$ or $E_{p}-E_{i s o}$ relations, among others. $^{2}$ Nevertheless, the different publications show that the constraints, on the cosmological parameters, using GRBs data are compatible with the results of supernovae Ia, which are currently the best standard candles. For a review of the current status of the standardization methods and details of the empirical relations, see references [15-17].

Recently, Demianski et al. [18] constructed a sample of GRBs using the luminosity distance of supernovae Ia to calibrate the correlation between the peak photon energy, $E_{p}$, and the isotropic equivalent radiated energy, $E_{i s o}$, of the GRBs (this is $E_{p}-E_{i s o}$ relation) and, consequently, construct a Hubble diagram of GRBs. The results of Demianski et al. [18] shows that the $E_{p}-E_{i s o}$ correlation does not have a significant dependence on redshift and, therefore, these GRBs data, in principle, can be used as a cosmological tool. How-

\footnotetext{
${ }^{1}$ Information about the project Euclid, see the web page: https://www. euclid-ec.org/ and on the LSST: https://www.lsst.org/.

2 The $\tau_{\text {lag }}-L$ is a correlation between spectrum lag and isotropic peak luminosity. The $V-L$ is a correlation between time variability and isotropic peak luminosity and the $E_{p}-E_{i s o}$ represents a tight correlation between the peak energy of spectrum and isotropic equivalent energy.
}

ever, this does not close the controversy, but we use these data because our goal here is not to do one research on observational constraints of precision on cosmological observables, rather to test a reconstruction methodology of $w(z)$ for high redshift. We use this calibrated sample for our analysis. The GRBs sample that we use consists of 162 measurements and covers a redshift interval between $0.03 \leq z \leq 9.3$ [18].

The theoretical distance module for the GRBs can be defined as follows:

$\mu_{t h}(z, \theta)=25+5 \log D_{L}(z, \theta)$,

where $\theta$ represents the parameters of a given cosmological model and $D_{L}$ represents the dimensionless luminosity distance. However, for our calculations, it is more convenient to rewrite this equation to determine the dimensionless comoving distance as follows ${ }^{3}$ :

$D_{c}=\frac{10^{\frac{\mu_{t h}-25}{5}}}{1+z}$.

In the above equation, we have used the fact that when the tricurvature is flat, the comoving distance is related to the luminosity distance by the relation: $D_{L}=(1+z) D_{c}$, and the comoving distance as a function of the Hubble parameter is defined by the following expression:

$D_{c}=\frac{c}{H_{0}} \int_{0}^{z} \frac{d x}{h(x, \theta)}$,

where $h(z, \theta)$ is the dimensionless Hubble parameter, $\frac{H(z)}{H_{0}}$. In our case, it is given explicitly by:

$$
\begin{aligned}
& h^{2}\left(z, \Omega_{m 0}, \Omega_{k}\right)=\left\{\Omega_{m 0}(1+z)^{3}+\Omega_{k}(1+z)^{2}\right. \\
& \left.+\left(1-\Omega_{m 0}-\Omega_{k}\right) \exp \left[3 \int_{0}^{z} \frac{1+w\left(z^{\prime}\right)}{1+z^{\prime}} \mathrm{d} z^{\prime}\right]\right\},
\end{aligned}
$$

where $\Omega_{m 0}$ and $\Omega_{k}$ represent the matter density parameter and curvature respectively. In this paper, we assume that $\Omega_{k}=0$, which matches the results of the Planck satellite $[6,7]$. To derive from the previous equation, an expression for EoS, is useful the definition [14,19,21],

$D_{c}=\frac{1}{\sqrt{-\Omega_{k}}} \sin \left(\sqrt{-\Omega_{k}} \int_{0}^{z} d z^{\prime} \frac{H_{0}}{H\left(z^{\prime}\right)}\right)$,

where we have included the term curvature. Therefore, we can use this equation above together with the equation of $h(z)$ to derive the equation of state as a function of $D_{c}[11]$ :

$$
\begin{aligned}
& w(z) \\
& =\frac{2(1+z)\left(1+\Omega_{k} D_{c}^{2}\right) D_{c}^{\prime \prime}-\left[(1+z)^{2} \Omega_{k} D_{c}^{\prime 2}+2(1+z) \Omega_{k} D_{c} D_{c}^{\prime}-3\left(1+\Omega_{k} D_{c}^{2}\right)\right] D_{c}^{\prime}}{3(1+z)^{2}\left[\Omega_{k}+(1+z) \Omega_{m}\right] D_{c}^{\prime 2}-\left(1+\Omega_{k} D_{c}^{2}\right) D_{c}^{\prime}}
\end{aligned}
$$

\footnotetext{
${ }^{3}$ The units of the equation two are given in $M p c$.
} 
Observing the previous equation, we can see that if we have observational data of the comoving distance and a method to reconstruct the derivatives of these data, then we can reconstruct the EoS directly from the observational data, except that we have to assume values for $\Omega_{m 0}$ and $\Omega_{k}$. This is a characteristic that always occurs when the EOS is reconstructed.

\subsection{The Sandage-Loeb effect: simulation of the hubble parameter}

In addition to the data of the GRBs, we use simulated data through the Sandage-Loeb effect (SL). The SL effect is a purely geometric measurement of the expansion rate of the universe. It can detect a change in the redshift of the source in the spectra of the Lyman- $\alpha$ forest of distant quasars in the range of $2 \leq z \leq 5$, also know as the "redshift desert". Initially, this effect was proposed by Sandage [22] in 1962. However, using the technology of the time, a temporary interval of the order of $10^{7}$ years was required to obtain an appreciable change in the redshift. Nevertheless, a reanalysis of the effect made by Loeb [23] relaunched the interest in this effect. Loeb argues that by using measurements from the Lyman- $\alpha$ forest of high-redshift quasars and with high-spectroscopic resolution associated with a 10-m class telescope, it would be possible to directly detect cosmic acceleration using time measurements of the order of $10^{1}$ years. Currently, the CODEX (COsmic Dynamics and EXo-earth experiment) [24], which is an optical high spectral resolution instrument and proposed for the European Extremely Large Telescope (E-ELT), can possibly detect this effect.

On the other hand, the effect of the atmosphere on experiments such as CODEX is strong and this is the reason why the measurements are planned to be obtained in the "redshift desert". As we are interested in reconstructing the equation of state at high redshift, we are going to use the CODEX prescriptions to simulate our data.

We can calculate the expected variation of the redshift of a chosen extragalactic source with time. If we assume that the source does not have any peculiar velocity, and so any peculiar acceleration, then it has a fixed comoving coordinate. In a homogenous and isotropic universe with a FLRW metric, consider that a source emits an electromagnetic wave during an interval $\left(t_{s}, t_{s}+\Delta t_{s}\right)$ and this wave is received by an observer during an interval $\left(t_{0}, t_{0}+\Delta t_{0}\right)$, then we can write [26]:

\footnotetext{
${ }^{4}$ Another possibility is to observe the sign of neutral hydrogen (HI) of galaxies in two different epochs. This experiment has been proposed for the SKA (Square Kilometre Array). In particular, the effect of the atmosphere is negligible on measures of the sign of the neutral hydrogen. This allows the SKA to make measurements for $z<1.65$ [25].
}

$\int_{t_{s}}^{t_{0}} \frac{d t}{a(t)}=\int_{t_{s}+\Delta t_{s}}^{t_{0}+\Delta t_{0}} \frac{d t}{a(t)}$

If we consider that $\Delta t_{s}, \Delta t_{0}<<t_{s}, t_{0}$, for example, for a typical light signal have an interval $10^{-14} s$, then the above expression leads to

$\frac{\Delta t_{0}}{a\left(t_{0}\right)} \approx \frac{\Delta t_{s}}{a\left(t_{s}\right)}$.

Considering that the redshift of the observed source is defined as $z\left(t_{0}\right)=\frac{\lambda_{0}-\lambda_{s}}{\lambda_{s}}$, we can arrive at the well-known expression $z\left(t_{0}\right)+1=\frac{a\left(t_{0}\right)}{a\left(t_{s}\right)}$. Thus, the source redshift changes for this interval of time can be written as

$\Delta z_{s}=z_{s}\left(t_{0}+\Delta t_{0}\right)-z_{s}\left(t_{0}\right) \equiv \frac{a\left(t_{0}+\Delta t_{0}\right)}{a\left(t_{s}+\Delta t_{s}\right)}-\frac{a\left(t_{0}\right)}{a\left(t_{s}\right)}$.

Expanding the first ratio on the right side of the equation above and considering only the first expansion order, we obtain:

$\Delta z_{s}=\left[\frac{\dot{a}\left(t_{0}\right)-\dot{a}\left(t_{s}\right)}{a\left(t_{s}\right)}\right] \Delta t_{0}$,

where the dot represents the time derivative. Thus, this equation can be rewritten as

$\Delta z_{s}=\left[H_{0}\left(1+z_{s}\left(t_{0}\right)\right)-H\left(t_{s}\right)\right] \Delta t_{0}$.

It is more convenient to express the redshift variation as a spectroscopic velocity shift as

$\Delta v \equiv \frac{c \Delta z_{s}}{1+z_{s}}=-H_{0} \Delta t_{0} c\left[1-\frac{h(z)}{1+z}\right]$,

where as previously, $h(z)=\frac{H(z)}{H_{0}}$. Therefore, if we can measure $\Delta z_{s}$ or equivalently $\Delta v$, then we can obtain a measure estimate of the Hubble parameter.

For a simulation of the Hubble parameter data using the SL effect, we used the $\Lambda C D M$ model as the fiducial model,

$h(z)=\sqrt{\Omega_{m 0}(1+z)^{3}+\Omega_{r 0}(1+z)^{4}+\left(1-\Omega_{m 0}-\Omega_{r 0}\right)}$,

where we have included a radiation component $\Omega_{r 0}$ and assume the values $H_{0}=70 \mathrm{~km} / \mathrm{s} / \mathrm{Mpc}$ and $\Delta t_{0}=10^{1}$ years.

The next step was to calculate the $\sigma_{\Delta v}$. To estimate the error, we use the prescription indicated by the collaboration CODEX [24], which establishes that the accuracy with which you can determine $\Delta v$ from the Lyman- $\alpha$ forest can be written as

$\sigma_{\Delta_{v}}=1.35\left(\frac{S / N}{2370}\right)^{-1}\left(\frac{N_{Q S O}}{30}\right)^{1 / 2}\left(\frac{1+z_{Q S O}}{5}\right)^{f} \frac{\mathrm{cm}}{\mathrm{s}}$,

where $S / N$ is the signal-to-noise ratio defined per $0.0125 \dot{A}$ pixel. $N_{Q S O}$ is the number of observed quasars, $z_{Q S O}$ represents their redshifts, $f=-1.7$ in the interval $2<z<4$ and 
$f=-0.9$ for $z>4$. The chosen number of quasars were 30 and the $S / N=3000$.

To determine the Hubble parameter, we must invert the equation to the spectroscopic velocity shift

$H(z)=\left[H_{0}-\frac{\Delta v}{c \Delta t_{0}}\right](1+z)$.

Since error propagation allows us to determine the uncertainty of $H(z)$ as

$\sigma_{H}=(1+z) \sqrt{\left(\frac{\sigma_{\Delta v}}{c \Delta t_{0}}\right)^{2}+\sigma_{H_{0}}^{2}}$

Therefore, as our simulated data are the Hubble parameter data, we need an expression that allows us to reconstruct the EoS, $w(z)$, directly from this observable. For such a case, it is possible to derive one expression directly from equation (4) as $[13,20]$

$w(z)=\frac{2(1+z) h h^{\prime}-3 h^{2}+\Omega_{k}(1+z)^{2}}{3\left[h^{2}-\Omega_{m 0}(1+z)^{3}-\Omega_{k}(1+z)^{2}\right]}$.

Again, it is interesting to note that this equation, as well as the previous equation for $w$, requires an external knowledge of the values of the $\Omega_{m 0}$ and $\Omega_{k}$ parameters and also in this case, by equation above, of the parameter $H_{0}$.

\subsection{Gaussian processes}

To perform the reconstruction of the equation of state, we use the non-parametric method of Gaussian processes, which is particularly important because it does not assume a specific model of the EoS. A Gaussian process can be written as:

$f(x) \sim G P(\mu(x), k(x, \tilde{x}))$,

where the value of $f$ when evaluated at a point $x$ is a Gaussian random variable with mean $\mu(x)$. Additionally, the value of the function $f$ at the point $x$ is not independent of the value of the function $f$ at some other point nearby $\tilde{x}$, but is related by the covariance function $k(x, \tilde{x})$. If consider observational data $\left(x_{i}, y_{i}\right)$ and assuming that the errors are gaussian and that $y_{i}=f\left(x_{i}\right) \pm \sigma_{i}$, where $\sigma_{i}$ are the $1 \sigma$ error bars and $i=1, \ldots, N$. We can to reconstruct the function $f$ at chosen points. This function is denoted by $f^{*}$. This reconstruction can be done through the joint distribution between $f^{*}$ and $y_{i}$. For details of the exact expressions see the reference [28]. In general, this reconstructed function has a reconstructed mean given by,

$\bar{f}^{*}(x)=\sum_{i, j=1}^{N} k\left(x, x_{i}\right)\left(M^{-1}\right)_{i, j}\left(f\left(x_{j}\right)-\mu(z)\right)$ in our reconstruction we chosen as prior mean function $\mu(z)$ a constant value and for the standard deviation:

$\sigma(x)=k(x, x)-\sum_{i, j=1}^{N} k\left(x, x_{i}\right)\left(M^{-1}\right)_{i, j} k\left(x_{j}, x\right)$

where $M_{i j}=k\left(x_{i}, x_{j}\right)+c_{i j}$ and $c_{i j}$ represents the covariance of the input data.

For our calculations, we assume the exponential function as a covariance function which is given by

$k(x, \tilde{x})=\sigma_{f}^{2} \exp \left(-\frac{(x-\tilde{x})^{2}}{2 \ell^{2}}\right)$,

where $\sigma_{f}$ and $\ell$ are called hyperparameters which are determined by maximizing the log marginal likelihood [27],

$$
\begin{aligned}
\ln = & -\frac{1}{2} \sum\left[f\left(x_{i}\right)-\mu\left(x_{i}\right)\right]\left(M^{-1}\right)_{i, j}\left[f\left(x_{j}\right)-\mu\left(x_{j}\right)\right] \\
& -\frac{1}{2} \ln |M|-\frac{1}{2} N \ln 2 \pi,
\end{aligned}
$$

where $M$ represent the determinant of $M_{i j}$. It is important to note that instead of optimizing these hyperparameters, they can be marginalized by using, for example, the MCMC method. However, a simple method of testing the validity of the optimization process is to vary the initial value of the hyperparameters and see if the values obtained change significantly. We have done this process and we determine that our hyperparameters do not change significantly. Therefore, we are confident in using the optimization process. On the other hand, the MCMC method should be used to obtain high precision restrictions associated with high quality data.

Additionally, this method allows to reconstruct the derivative of the data. To implement this method, we use the public package GaPP [28]. For details on the statistical method, reference [27] may be useful and for applications in cosmology, consider reference [13].

\subsection{Methodology}

In an illustrative way, our methodology is summarized in Fig. 1. Initially, we begin with observational data or simulated data. These data are analyzed using a non-parametric statistical method. As a result of this statistical analysis, we obtain, as in our case, the comoving distance $D_{c}$ and its derivatives $D_{c}^{\prime}$ and $D_{c}^{\prime \prime}$ and the Hubble parameter $h$ and its derivative $h^{\prime}$. Then, we use a model-independent approach to reconstruct the EoS. To estimate the errors of the reconstruction, we use the Monte Carlo method.

This methodology is quite general because we can assume other non-parametric methods in addition to the Gaussian process method, such as the principle component analysis [29-32] or, as in reference [33], a non-parametric method that consists of a combination of the Loess and Simex methods. Analogously, another model-independent approach can be 
Fig. 1 Schematic summary of the methodology used

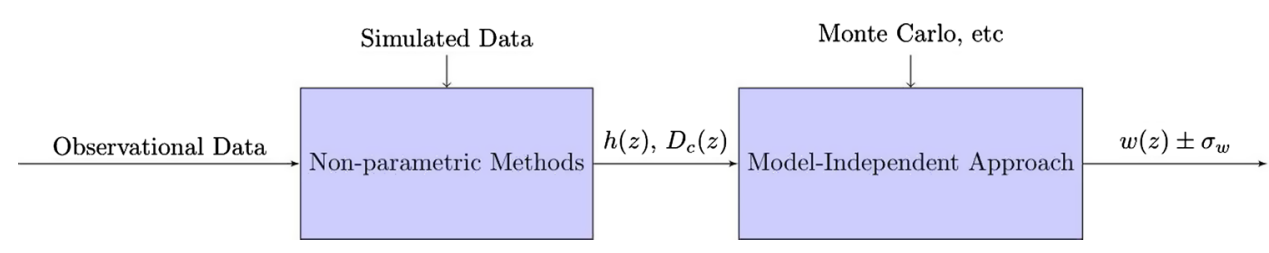

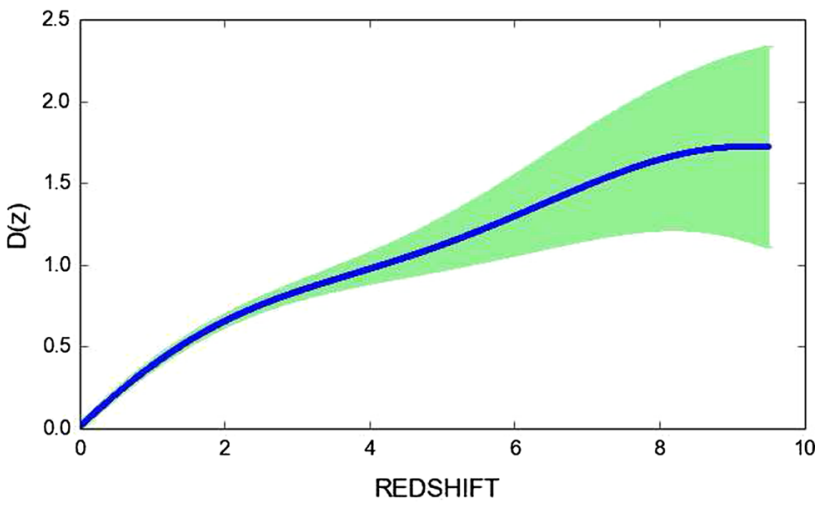

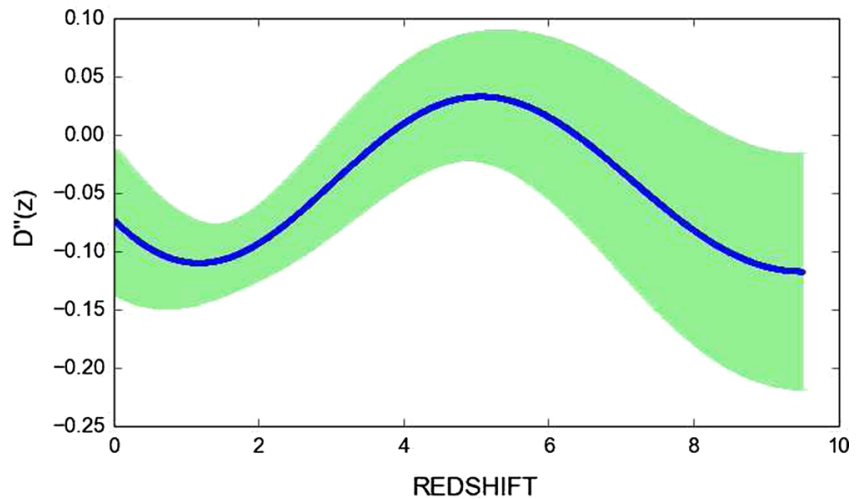

Fig. 2 On the left-hand plot, we shown the 162 data of the GRBs for the comoving distance. In the other plots, we show the reconstruction of this observable and its derivatives

assumed. For instance, we can assume a consistency test of the $\Lambda C D M[11]$ or we can use the cosmographic parameters [26]. To estimate reconstruction errors, an alternative to the Monte Carlo method is the method of resampling, such as the jackknife method [34], among others.

\section{Results}

In Fig. 2, we see the results of the reconstruction of the comoving distance and its derivatives using the GRBs data, following the methodology presented in Sect. 2. The central blue line represents the average reconstruction value, which is reconstructed with $1 \sigma$. In Fig. 3, we see the complete reconstruction of the EoS. To reconstruct the function $w(z)$ we follow the prescriptions of Sect. 2.3 of the reference [28]. The values and errors of the $w(z)$ function are determined by Monte Carlo sampling. In each calculation the functions $D_{c}, D_{c}^{\prime}, D_{c}^{\prime \prime}$, etc, are drawn from a normal multivariate dis- tribution. This distribution has as components of the mean vector the functions $\left(D_{c}, D_{c}^{\prime}, D_{c}^{\prime \prime}\right)$ and $\left(h, h^{\prime}\right)$ reconstructed using GP. As covariance matrix the reconstructed covariances between the functions and their derivatives $\operatorname{cov}\left(D_{c}, D_{c}^{\prime}, D_{c}^{\prime \prime}\right)$ and $\operatorname{cov}\left(h, h^{\prime}\right)$ using the GaPP. We perform around 100,000 chains at each redshift point where the functions were reconstructed and assume a specific value of $\Omega_{m 0}$. As observed, the errors increase significantly for high redshifts, especially for values $z>2.0$. The central blue line represents the average reconstruction value. In Fig. 4, we present the effects of changing the value of $\Omega_{m 0}$. We see that the effect is very small. What we can emphasize is that in all the cases, for high redshift, we observe a tendency of the average value of $w(z)$ for a universe dominated by matter.

In general terms, we can say that the EoS reconstructed with one and two sigmas shows a fairly large error propagation and it does not allow us to distinguish between cosmological models. However, we have proved that even with large error propagation, it is possible to reconstruct the EoS. 
Fig. 3 The cosmic equation of state using the reconstructed data of the comoving distance by the model-independent approach is provided by Eq. (6). determined by Monte Carlo. The reconstruction is done for the intervals: $0.03 \leq z \leq 9.3$. We can see that for redshift greater than $z>3$, the errors increase noticeably. The dependence on the value of the parameter $\Omega_{m 0}$ for high redshift are covered by the large errors. We use $\Omega_{m 0}=0.315 \pm 0.007$ $[6,7]$ and flatness The calculation of uncertainty is
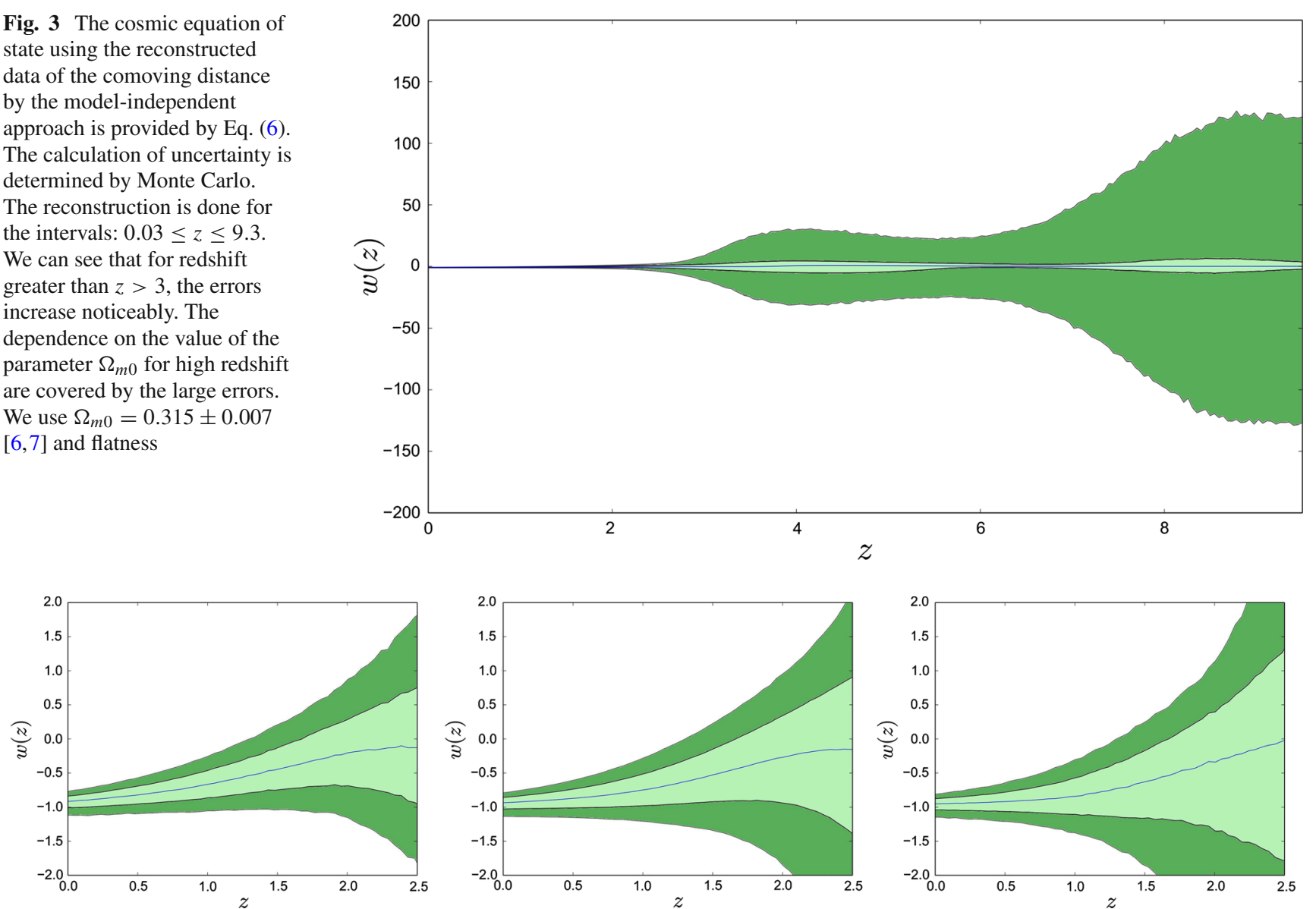

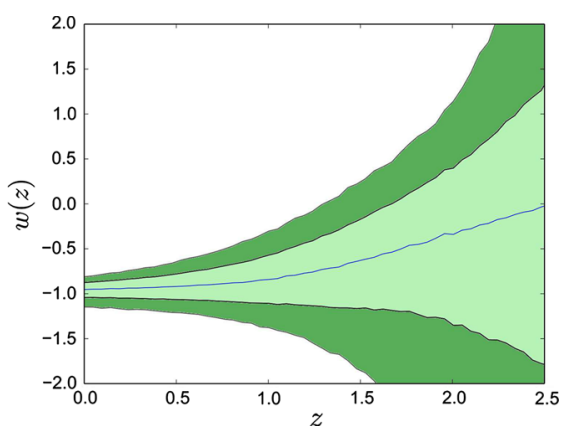

Fig. 4 We show the dependency of the reconstruction of the state equation with the value of $\Omega_{m 0}$. This dependence is evident for minor redshifts that are depicted in the 3 figures. In the left, $\Omega_{m 0}=0.200$; in the centre, $\Omega_{m 0}=0.275$ and; to the right, $\Omega_{m 0}=0.400$

This same methodology could be used when the number and quality of the GRBs data increases considerably.

In Fig. 5, we can see the result of applying the methodology of the section II.B. We presented the simulated data for the function $h(z)$ and its derivative $h^{\prime}(z)$ in a redshift between $2-5$ using the Gaussian processes. The simulated errors are relatively small when compared to the result of the GRBs. For example, compare the vertical scale between Fig. 3. and the reconstruction shown in the lower right section of Fig. 5. Although they are not real data, we hope that future data will follow this trend and that robust constraints on the observables can be determined.

On the other hand, given that the SL effect depends on the assumed value of $H_{0}$, we considered the dependence of changing this value in the reconstruction of $w(z)$. Since the errors are still large, the effect of $H_{0}$ on the EoS is quite negligible. We used the values of $H_{0}=73.24 \pm 1.74$ $\mathrm{km} / \mathrm{s} / \mathrm{Mpc}$ as measured for Supernovae Ia $[35,36]$ and the value $H_{0}=67.51 \pm 0.64 \mathrm{~km} / \mathrm{s} / \mathrm{Mpc}$ as extracted from Planck 2015 TT, TE, EE + lowP + lensing data [6,7].

\section{Conclusions}

We performed a reconstruction of the EoS using Gaussian processes with GRBs data as well as a model-independent approach. The analysis was carried out by considering different values of the $\Omega_{m 0}$, which covers the current observation limits. Moreover, we also use the SL effect to simulate the Hubble parameter data in the so-called "redshift desert" using the flat $\Lambda C D M$ model as a fiducial method with values: $\Omega_{m 0}=0.275$ and $H_{0}=70 \mathrm{~km} / \mathrm{s} / \mathrm{Mpc}$. The reconstruction using the simulated data is significantly better (lower propagation of uncertainty) than those determined by the GRBs data. However, these measures are strongly dependent on the underlying model.

The fact that our reconstruction is independent of the assumed cosmological model allows us to observe that in general terms, the form of the reconstruction of $w(z)$ must be associated with a continuous function and good behavior, without sudden jumps. Further, it can be seen that the EoS for high redshift is compatible with a phase dominated by matter (as expected by theory). However, with the current 

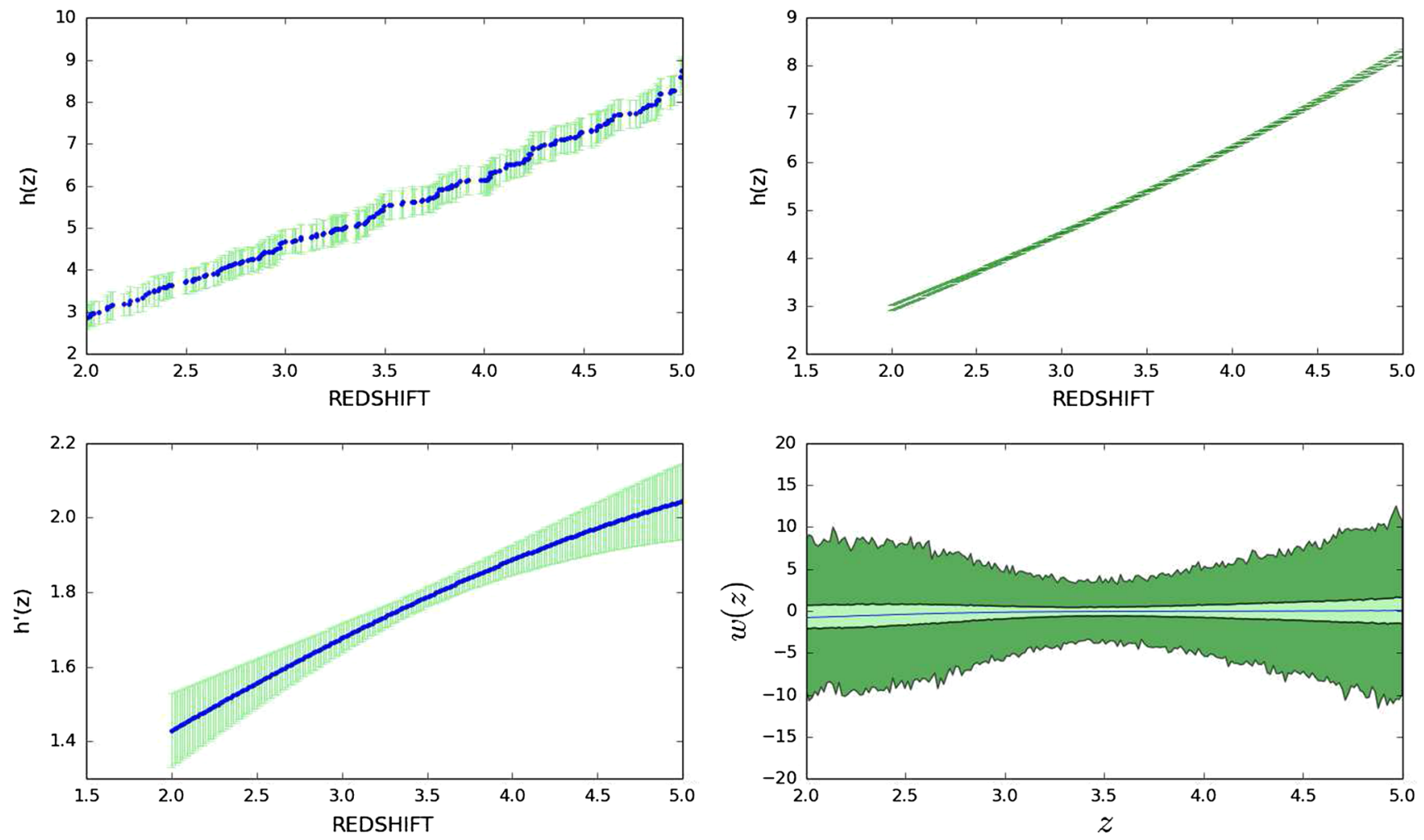

Fig. 5 In the top, we can see the simulated data using the Sandage-Loeb effect and the reconstruction of $h(z)$ using the Gaussian process. In the bottom, we see the reconstruction of the first derivative $h^{\prime}(z)$ and the forecasted constraints for the cosmic equation of state with $\Omega_{m 0}=0.315 \pm 0.007$ $[6,7]$

data, it is not possible to specify exactly where this happens, i.e., where $w(z)=0$, due to the uncertainty.

In general, to perform a reconstruction of cosmological observables for high redshift, it is necessary to improve the conjunction between statistical methods and cosmology. In this study, we have presented specific cases. However, other combinations of statistical methods along with independent approximations can be studied. A good calibration of this methodology will allow us to use future data to reconstruct the EoS for high redshifts with robustness and high precision, which will help discriminate between competitive cosmological models.

Acknowledgements J.C.F acknowledges financial support from FAPES and CNPq. M. M. M acknowledges the support from FAPES. A. M. V. T would like to dedicate this research to celebrate the scientific career of Ioav Waga, my PhD advisor who has recently retired from UFRJ.

Data Availability Statement This manuscript has no associated data or the data will not be deposited. [Authors' comment: The data used in this article have been published in the reference [18] M. Demianski, E. Piedipalumbo, D. Sawant and L. Amati, A \& A.598 (2017), A112.]

Open Access This article is licensed under a Creative Commons Attribution 4.0 International License, which permits use, sharing, adaptation, distribution and reproduction in any medium or format, as long as you give appropriate credit to the original author(s) and the source, provide a link to the Creative Commons licence, and indicate if changes were made.The images or other third party material in this article are included in the article's Creative Commons licence, unless indicated otherwise in a credit line to the material. If material is not included in the article's Creative Commons licence and your intended use is not permitted by statutory regulation or exceeds the permitted use, you will need to obtain permission directly from the copyright holder. To view a copy of this licence, visit http://creativecomm ons.org/licenses/by/4.0/.

Funded by $\mathrm{SCOAP}^{3}$.

\section{References}

1. P.J.E. Peebles, B. Ratra, Rev. Mod. Phys. 75, 559 (2003)

2. M.J. Mortonson, D.H. Weinberg, M. White, Chin. Phys. C 38, 361 (2015)

3. T. Clifton, P.G. Ferreira, A. Padilla, C. Skordis, Phys. Rep. 513, 1 (2012)

4. Y. Susuki et al., ApJ. 746, 85 (2012)

5. M. Kowalski et al., ApJ. 686, 749 (2008)

6. N. Aghanim et al. [Planck Collaboration]. arXiv:1807.06209

7. P.A.R. Ade, et al. [Planck Collaboration], A\&A 594, A13 (2016)

8. J. Prez-Romero, S. Nesseris, Phys. Rev. D 97(2), 023525 (2018)

9. F. Anagnostopoulos, S. Basilakos, Phys. Rev. D 97, 063503 (2018)

10. M. Kunz, S. Nesseris, I. Sawicki, Phys. Rev. D 94(2), 023510 (2016)

11. C. Zunckel, C. Clarkson, Phys. Rev. Lett. 101, 181301 (2008)

12. M. Seikel, C. Clarkson, M. Smith, JCAP 06, 036 (2012) 
13. M. Seikel, S. Yahya, R. Maartens, C. Clarkson, Phys. Rev. D 86, $083001(2012)$

14. D. Huterer, M.S. Turner, Phys. Rev. D 60, 081301 (1999)

15. S. Basilakos, L. Perivolaropoulos, Mon. Not. R. Astron. Soc. 391, 411 (2008)

16. F.Y. Wang, Z.G. Dai, E.W. Liang, New Astron. Rev. 67, 1 (2015)

17. H.-N. Lin, X. Li, Z. Chang, Mon. Not. R. Astron. Soc. 455, 2131 (2016)

18. M. Demianski, E. Piedipalumbo, D. Sawant, L. Amati, A\&A 598, A112 (2017)

19. A.A. Starobinsky, JETP Lett. 68, 757 (1998)

20. D. Huterer, M.S. Turner, Phys. Rev. D 64, 123527 (2001)

21. T. Nakamura, T. Chiba, Mon. Not. R. Astron. Soc. 306, 696 (1999)

22. A. Sandage, Astrophys. J. 136, 319 (1962)

23. A. Loeb, Astrophys. J. 499, L111 (1998)

24. J. Liske et al., Mon. Not. R. Astron. Soc. 386, 1192 (2008)

25. H.-R. Klockner, D. Obreschkow, C.J.A.P. Martins, A. Raccanelli, D. Champion, A.L. Roy, A. Lobanov, J. Wagner, R. Keller, Proceedings, Advancing Astrophysics with the Square Kilometre Array (AASKA14), PoS AASKA14, vol. 027 (2015). arXiv:1501.03822 [astroph.CO]
26. S. Weinberg, Gravitation and Cosmology (Wiley, New York, 1972), p. 416

27. C. Rasmussen, C. Williams, Gaussian Processes for Machine Learning (The MIT Press, Cambridge, 2006)

28. M. Seikel, C. Clarkson, M. Smith, JCAP 06, 036 (2012)

29. G.B. Zhao, L. Pogosian, A. Silvestri, J. Zylberberg, Phys. Rev. Lett. 103, 241301 (2009)

30. S. Nesseris, D. Sapone, Phys. Rev. D 90, 063006 (2014)

31. R.G. Crittenden, L. Pogosian, G.B. Zhao, JCAP 0912, 025 (2009)

32. C. Clarkson, C. Zunckel, Phys. Rev. Lett. 104, 211301 (2010)

33. C. Escamilla-Rivera, J.C. Fabris, Galaxies 4, 76 (2016). arXiv:1511.07066

34. M. Shirasaki, M. Takada, H. Miyatake et al., Mon. Not. R. Astron. Soc. 470, 3476 (2017)

35. A.G. Riess et al., Astrophys. J. 826, 56 (2016)

36. A. G. Riess, et al., Astrophys. J. 861, 126 (2018). arxiv:1804.10655 